\title{
Ultra-High Dilutions and Homeopathy: Can They Be Explained without Non-Local Theory?
}

\author{
Yannis Almirantis ${ }^{1}$ Konstantinos Tsitinidis ${ }^{2}$ \\ ${ }^{1}$ Institute of Biosciences and Applications, National Centre for \\ Scientific Research 'Demokritos', Athens, Greece \\ 2 'Homeopathie', Homeopathy Centre, Athens, Greece
}

\begin{abstract}
Address for correspondence Yannis Almirantis, PhD, Institute of Biosciences and Applications, National Centre for Scientific Research 'Demokritos', 15310 Athens, Greece (e-mail: yalmir@bio.demokritos.gr).
\end{abstract}

\author{
Abstract \\ Keywords \\ - homeopathy \\ - memory of water \\ - potentised ultra- \\ diluted remedies \\ - Benveniste's \\ experiment \\ - non-locality \\ - generalised \\ entanglement \\ - causality \\ - similia principle
}

We discuss questions related to the 'Benveniste Affair', its consequences and broader issues in an attempt to understand homeopathy. Specifically, we address the following points:

1. The relationship between the experiments conducted by Benveniste, Montagnier, their collaborators and groups that independently tested their results, and 'traditional' homeopathy.

2. Possible non-local components such as 'generalised entanglement' as the basis of the homeopathic phenomenon and experimental evidence for them.

3. The capability of highly diluted homeopathic remedies to provoke tangible biological changes in whole organisms and cellular experimental systems.

4. Aspects of the similia principle related to the above.

5. Suggestions that can lead to experimental verifications of the non-local hypothesis in homeopathy.

\section{Introduction}

In this article, we do not attempt to overview the whole history of the work of Jacques Benveniste's team that appeared in the 1980 s and of the subsequent evolution of their ideas that resulted in the concept of digital biology. There are already other articles ${ }^{1-4}$ dedicated to this historical account, some of which written by protagonists of this endeavour, as well as entire books. ${ }^{5,6}$ The purpose of the present article is mainly twofold, with its two objectives strongly interconnected. First, to point out that Benveniste and his followers' work constitutes an integral part of the field of homeopathy, as it is motivated by homeopathic medicine and it may serve to shed light on the foundation of the homeopathic phenomenon. Second, to present evidence that the combined corpus of data from the line of research of Benveniste, the rest of the experimental work on ultra-high dilutions (UHDs) and homeopathic medicine, cannot be understood outside a non-local approach, in the spirit described by Walach, ${ }^{7-9}$ Milgrom, ${ }^{10-12}$ Weingärtner, ${ }^{13-15}$ Beauvais $^{16-18}$ and other investigators.
The opinion that the experiments of Jacques Benveniste ${ }^{19}$ were erroneous or ill-founded may be encountered even among people from the homeopathic community. ${ }^{20}$ However, we believe that the verification of these experiments by Jean Sainte-Laudy, Madeleine Ennis and others, ${ }^{21,22}$ despite the subsequent equivocal results of different scientific teams in their attempts to replicate that outcome, renders the picture very complex, as well as open to alternative explanatory hypotheses. First, we have to note that the research groups of Benveniste and Ennis already had an outstanding reputation and record in the scientific community. Additionally, after the disastrous impact of the invalidation of Benveniste's results on his reputation by the Nature team ${ }^{23}$ which was mainly oriented towards the detection of scientific fraud-any research group attempting the same kind of experimentation would obviously have been well aware of the risk involved. Especially in the case when Benveniste's results were successfully replicated, the obtained outcome would have to be exceptionally well checked. We make these comments here because we believe that a simple rejection of received

June 1, 2017

accepted after revision

April 12, 2018

published online

June 5, 2018
Copyright $\odot 2018$ The Faculty of Homeopathy 
the results of Benveniste, Ennis and others might deprive us from insights into the epistemological particularities of the field of potentised ultra-diluted remedies.

It is beyond the scope of the present article to comment systematically on differences between the used experimental models, so we present Sainte-Laudy's and Ennis's experiments simply as a continuation of Benveniste's work as we mainly focus on the existence of observable effects when working with UHDs in general. Nevertheless, they used different systems: the inhibitory effect of high dilutions of histamine on basophil activation (Ennis), and the direct activation of degranulation of basophils by high dilutions of anti-immunoglobulin $\mathrm{E}$ ( $\mathrm{IgE}$ ) (Benveniste), as is reviewed in detail elsewhere. ${ }^{3,24}$

\section{The Emergence of a Non-Local Explanatory Framework in Homeopathy and in Benveniste's Experiments}

Several authors have proposed that both homeopathy and experimentation with (potentised by succussion) UHDs might involve non-local aspects of nature, in the spirit of non-locality found in quantum mechanics ${ }^{25}$; see also references given in the introduction. The principal motive for the search for connections between these remote scientific fields, which might appear unorthodox, has been the observation of an elusive aspect ${ }^{7,26}$ in homeopathic medical practice and in experimentation with UHDs. This elusiveness becomes particularly evident since repeatability is clearly weak. This weak repeatability, along with some other features of homeopathic medicine, are landmarks indicating that here we might not simply have experimental errors. A tendency of errors to occur within an experimental procedure, or even the existence of phenomena with an inherently stochastic nature, raises the need for the application of suitable statistics for the assessment of eventually positive results. This is not unusual and may be dealt with within the current biomolecular explanatory framework. Instead, there is growing evidence that in the fields we examine here, we witness the appearance of epistemologically novel features: to use the terminology of T. S. Kuhn, we see the emergence of a new paradigm. ${ }^{27}$ It is very premature to fully describe this new scientific realm, but the 'weak quantum theory' as formulated by Atmanspacher et al, ${ }^{25}$ theoretical work by Beauvais, ${ }^{17,18}$ and a few other attempts, might represent previews of what its principal components are.

Within this line of thought, Harald Walach suggested that the often poor outcome of double-blind clinical trials (DBCTs) in assessing homeopathy might be a consequence of an impossibility of UHDs to convey information about their initial solute. Reasons for this are paradoxes and contradictions created by constraints due to special relativity. ${ }^{9}$ To test the non-local hypothesis for homeopathy, Walach et al and Möllinger et $\mathrm{al}^{28-30}$ conducted specially designed blinded pathogenetic trials (provings) where they observed phenomena incompatible with local and (conventionally) causal explanatory schemes for homeopathy. Thus, they verified preliminary ${ }^{31}$ and anecdotal ${ }^{7,30}$ observations point- ing towards a 'smearing' of the remedy effect in both DBCTs (across verum and placebo group of patients) and blinded provings (across provers receiving remedy or placebo treatment). One completely blinded study, ${ }^{29}$ with two groups of provers taking different remedies and one group taking placebo, led to statistically significant 'paradoxical' results, such as finding symptoms in a group typical of the remedy given to the other group, and symptoms typical of a remedy found in a group that only later received it: that is, a kind of 'presentiment' effect. According to Walach, if such effects are treated causally, 'they go away, change channel or do something crazy'. ${ }^{9}$ As suggested by Beauvais, finding symptoms typical of one remedy in another study group may represent such a 'channel change' due to the inadequacy of non-local correlations for the transmission of information. ${ }^{17}$

In homeopathy, and especially in view of some key properties of remedies, a direct implication of contextuality and significance appears. This becomes particularly evident when we take into account the 'coincidence' of the symbolic meaning of several homeopathic remedies with their curative specificity, as we commented in an earlier work. ${ }^{32}$ Examples are remedies such as Aurum met, Plumbum met (with their symbolism provided by mythology), Platina (in its social connotation) or Carbo vegetabilis (on the basis of its traditional manufacturing). Such features of homeopathy are entirely inexplicable on the basis of any biomolecular or more generally conventional explanation, which does not imply some extended form of causality directly involving significances.

The link of Benveniste's project to homeopathy is clearly evidenced in several accounts: see articles by Thomas ${ }^{2}$ and Poitevin. ${ }^{3}$ As Poitevin comments, 'it is clear that the work about "memory of water" was born of the will to study the biological effect of homeopathic medicines'. Moreover, Poitevin specifies that although Benveniste's team had previously carried out experiments with a homeopathically prepared UHD of bee venom, the experiment that they chose to present in the Nature publication was a variation involving the direct activation of basophils by anti-IgE. Poitevin mentions that the reasons for this choice were complex: technical, strategic as well as psychological. ${ }^{3}$ However, the most substantial link between homeopathy and Benveniste's project becomes apparent when we take into account a main feature they share, which generally is considered a serious flaw of both research fields: namely their elusive aspect. This, in the case of Benveniste's endeavour, might be at the origin of the notorious lack of consistency in the results obtained during attempts to replicate the initial experiment published in Nature and its subsequent variations.

The cumulative results from 886 experiments, both blinded and open, conducted by Benveniste's group over almost a decade, have been presented and analysed by Beauvais. $^{6,16,33}$ These experiments, pertaining to the field named by Benveniste as 'digital biology', concern the transmission of the activity of a potentised solution of a pharmacological agent to pure water through an electromagnetic signal and then testing if this transmission did in fact occur, by means of an isolated heart model, using the 'Langendorff 
apparatus'. Interestingly, this analysis shows a striking difference between blinded and open, but also between 'internally' and 'externally' blinded experiments: that is, experiments where the blinding was performed either by a member of the team or by an observer unrelated to this research group (see discussion and Table 2 within reference $^{16}$ ). It appears that a contextual factor is of paramount importance for the success of these experiments. In brief, the open experiments and those blinded by a member of the group were successful with high statistical significance: that is, the response of the animal heart was consistent with the use either of 'informed' or of 'naïve' water (exposed to the electromagnetic signals of the active solution or not, respectively). In contrast to that, experiments blinded by scientists unrelated to the experimenters' group systematically failed to distinguish the identity of the tested sample.

Inspired by the difference between 'internally' and 'externally' blinded experiments described above, and taking into account the drop in efficacy of homeopathy when performed in blinded conditions, ${ }^{26}$ Francis Beauvais ${ }^{17}$ developed a quantum-like model for the study of probabilities in DBCTs. Beauvais showed a parallelism between single-photon interference experiments and randomised placebo-controlled homeopathy trials. He proposed specific modifications of the DBCT procedure (which he named in situ randomisation and unblinding), which could suppress the smearing effect occurring between verum and placebo group. Most importantly, an application of Beauvais' suggestion in a plant model has been presented in a recent publication, ${ }^{34}$ where the smearing effect has indeed diminished. If this finding was corroborated by further experimentation, the non-local nature of homeopathy, and of experiments involving ultradiluted potentised solutions as well, would be convincingly illustrated.

The fact that features observed in Benveniste's experimental work led to a suggestion of improvements in the practice of blinded clinical trials in homeopathy corroborates the existence of common ground between these two fields.

\section{Mind-Matter Interactions in Homeopathy and Benveniste's Experiments}

As Walach has commented, the elusive aspect and several specific features of the clinical testing of homeopathy and of the associated experimental work remind one of characteristics commonly observed in the field of parapsychology. ${ }^{8}$ Another link between the domain of the study of mindmatter interaction and homeopathy stems from a highly automated attempt to repeat an experiment (suggested by the Benveniste group) by an independent team of experts. This attempt was requested by the United States Defense Advanced Research Projects Agency (DARPA). The details of this work were published in the FASEB Journal. ${ }^{35}$ There, in the final experiment conducted in the absence of any member of the French team, no biological effect was found to be transmitted through electromagnetic signals-as had been the case with the initial observation of Benveniste's group. Thus, the result of the attempt of the DARPA team to replicate
Benveniste's experiment was declared negative. However, in preliminary repetitions of the experiment where a member of the French team participated, such transmission appeared!

This 'experimenter effect' was extremely strange, as the aforementioned publication stresses that no activity took place on the part of Benveniste's collaborator in this experiment that might have influenced the result or violated the research protocol, including blinding and randomisation. Perhaps, this type of experiment, which lends itself to full automation, represents a suitable tool for the study of aspects of mind-matter interaction. It may be seen as a case of generalised entanglement between a person and an object, as in the entanglement among patient-practitionerremedy, suggested by L. Milgrom, ${ }^{10}$ or of variations of it, ${ }^{7}$ conjectured to occur in homeopathic medical practice.

The experimenter effect and the related features of homeopathic therapeutics, which we have mentioned thus far, might correspond with the 'role of the observer' in quantum mechanics, and with aspects of the EinsteinPodolsky-Rosen (EPR) paradox and its implementations, which have led to the conclusion that nature as described by quantum mechanics is non-local. ${ }^{36}$ However, the authors who formulated the non-local hypothesis for homeopathy do not suggest that experimentation with UHDs, digital biology and homeopathy constitute macroscopic quantum mechanics. They contended that here a novel situation presents itself where some (but not all) of the features of the quantum microcosm reappear, ${ }^{8,14,25}$ where quantum mechanics may serve as a metaphor for homeopathy, ${ }^{10}$ and where new tools, such as contextuality and entanglement, may be sourced from the quantum formalism. ${ }^{14,17}$

Moreover, the relationship between the symbolic meaning of a crude substance and the curative properties of the corresponding remedy, along with the identification of the curative action of remedies either in a direct or an inverse sense with respect to the crude substance-in a way reminiscent of similar patterns encountered in linguistics, myths and dream analysis ${ }^{32}$-poses additional challenges to any attempts to fully understand the underlying phenomenon. Metaphors from quantum mechanics have been of great importance for us to realise just how fundamental the role of non-repeatability is for the understanding of the whole homeopathic phenomenon and the recognition of the eventual crystallisation of a new scientific paradigm. This holds true for research in the field of UHDs as well. Further clinical, experimental and theoretical work for a clearer description of this new domain is certainly needed.

A further question might arise here, concerning the relation between the formalistic description of the homeopathic phenomenon (inspired by quantum mechanics) and the conjectured experimenter effect, or in broader terms any mind-matter interactions. Is their relation inclusive? Could we say that the quantum-like formalism describes the general modalities, while individuals involved are represented by, say, the values of suitable coefficients? Are things as simple as that or is the quantum metaphor not able quantitatively to describe the experimenter effect? And in that case, 
can features deduced from the application of the formalism be interwoven with personal involvement? Poitevin points out experimenter effect even in some of the initial work of Benveniste's group, ${ }^{4,37}$ while in the DARPA report a testimony by Benveniste himself is included, stating that 'he observed similar experimenter variability in his laboratory' and that 'certain individuals consistently get digital effects and other individuals get no effects or block those effects. ${ }^{\text {, }}{ }^{3}$ The relation between any formalistic description and mindmatter interaction may continue to challenge our ideas about causality for a long time to come.

Fisher comments on an apparent difficulty of most theories involving generalised entanglement to account for selftreatment with homeopathy. ${ }^{38}$ We suggest that perhaps this should be viewed in the way Lacanian psychoanalysis considers the collective paternal role in society through the use of language, the so-called Name-of-the-Father, instead of the true, physical father in its role in the development of the Oedipus complex. ${ }^{39}$ The homeopathic community as a whole elaborates on the meaning of remedies, and the interaction of the patient with this knowledge provides him/her with the ability of self-prescription (which Milgrom has named 'Phoning a friend ${ }^{11}$ ). Taking generalised entanglement more literally, we might contend that here mind-matter interaction is present, in parallel with and indistinguishable from conventional causal ways of transmission of knowledge. Thus, we could say that any correct prescription of a remedy does involve the collective experience and knowledge of the entire homeopathic community, activating generalised entanglement as a curative means.

\section{The Compatibility between Benveniste's Experiments and Homeopathy}

In a recent article referring to the Benveniste case, ${ }^{20}$ Vithoulkas stated that, from the very beginning, he considered Benveniste's attempts for in vitro experimentation with UHDs incompatible with the principles of homeopathy. A main point in Vithoulkas' objection is: 'A high potency can never bring about a structural change in the organism, like the one Benveniste claimed'. The term 'structural' here is somewhat restrictive but, if we replace it with 'physiological' or simply 'biological', the homeopathic treatment of patients with high potency remedies provides plenty of such examples. Needless to say, some of them clearly imply structural changes in the affected tissues and cells, and in experimentation with cell cultures the only expected effects are 'structural'-and in any case 'observable'-changes such as the ones found in Benveniste's experimentation. Also, Vithoulkas posits that basophil degranulation could never have been observed as a result of the action of a homeopathic preparation of bee venom (or, in the experiment published in Nature, of anti-IgE). He bases this claim on the conviction that a homeopathic remedy always cures symptoms that are produced by the substance which is at the basis of this remedy ('the mother tincture' or more generally 'the crude substance').

However, homeopaths know at least one situation where homeopathic remedies often produce the same type of effects as the substance used for their preparation: their administration to healthy individuals during the proving of the remedy, where the observed symptoms of the tested potentised remedy often largely coincide with the symptoms caused by the crude substance. Generally, the administration of a remedy, either for proving or for therapeutic purposes, may give rise to a complex, possibly non-monotonic, response of the organism, with reactions conditioned by the personal history and sensitivity of the prover or the patient. Oscillatory responses to a remedy have also been observed. ${ }^{40}$ In Benveniste's article in Nature, ${ }^{19}$ an oscillatory response of the intensity of basophil degranulation is observed, in this case not over time but depending on the degree of dilution of the utilised anti-IgE. In experiments with Arnica montana acting on a human macrophage cell line, where its effect on gene expression was measured and when low and high potency dilutions were used, patterns of down- and up-regulation of several genes were observed correspondingly. This situation could be characterised as a 'hormetic' effect. ${ }^{41}$ Non-monotonic and hormesis-like responses, as well as oscillations, are indications of the highly non-linear and complex character of organisms and cellular systems, and very probably of the homeopathic phenomenon itself. ${ }^{42}$

On closer examination of the similia principle itself, claimed by Vithoulkas as contrary to the whole concept behind Benveniste's experiment, ${ }^{20}$ it is well known that several homeopathic remedies (Chamomilla, Arnica, Hypericum, to cite only a few) cure the same ailments or symptoms as those for which the mother tinctures (used in their preparation) are used. The formulation of the similia principle verified by homeopathic experience is: 'The more complete is the similarity between a patient's symptoms and the remedy picture during homeopathic pathogenetic trials (HPTs, provings), the more effective the remedy will be when prescribed to this patient'. This formulation states that similarity is observed between symptoms caused or alleviated by potentised solutions in both cases (provings and prescription to a patient). We know that in several cases this distinction is not made in the homeopathic literature and this entails the risk of exposing homeopathy to undue criticism. For example, believing that chamomile infusion might cause colic spasms or convulsions would be against the experience of any doctor.

In fact, all three of the following cases may be encountered in Materia Medica: (1) the standard case where the homeopathic remedy cures symptoms caused by the corresponding crude substance, (2) the inverse case, where the homeopathic remedy cures symptoms that the corresponding crude substance also cures, and (3) remedies whose crude substance causes symptoms unrelated to the curative spectrum of the remedy or is mostly inert from a medical point of view. These points have been discussed in greater detail in a previous publication by one of the present authors. ${ }^{32}$ We only have to add that most of the cases where the curative spectrum of a substance and of the corresponding remedy is the same are natural medicines and not venoms as in the experiment of basophil degranulation. Even so, we cannot 
invalidate the outcome of Benveniste's work as contradicting any main principle of homeopathy, because, as discussed above, such fundamental contradictions do not exist.

\section{Continuation of Benveniste's Endeavour by Luc Montagnier: Possible Links with the Theoretical Framework Involving Non- Locality and Generalised Entanglement}

Thus far, we have mentioned only basophil degranulation and related experiments by Benveniste and collaborators in the field they termed digital biology. Experimental work by the Nobel laureate Luc Montagnier builds on Benveniste's last years of investigation that dealt with electromagnetic signals emitted by potentised solutions. Montagnier et al verified this property for bacterial DNA as well. ${ }^{43}$ More unexpectedly, they carried out experiments where pure water had been exposed to signals emitted by potentised solutions of DNA and pointed out that the DNA sequence information may be transmitted in this way. They used this water as solvent and, without a DNA template, they synthesised the specific DNA sequence through polymerase chain reaction $(P C R) .{ }^{44,45}$

Montagnier's work was heavily criticised, yet a number of experts from several countries collaborated with him and verified the originality of the reported results. We firmly believe that the only way to broaden our present understanding of the workings of nature is by attempts, free from any prejudice, at reproduction and further study, by independent research groups to verify any reported unexpected or extraordinary findings. Otherwise, we risk transforming our present scientific convictions to nearreligious dogmas. We have to keep in mind that nonclassical causality in homeopathy might apply in Montagnier's experiments too, as they also imply paradoxical properties in the absence of the initial solute. If this is the case, the type of statistical behaviour of the outcome of such experiments is yet to be determined. We have not found any published results of this team differentiating between blinded and non-blinded experiments, let alone between internally and externally blinded ones. We suggest that the production of such results for the work of Montagnier is of paramount importance.

In this case, samples of naïve water should be included in the tests; both open and blinded. The distributional features of the 'failed' experiments would indicate whether PCR without the DNA template distinguishes between blinded and non-blinded repetitions, and between internally and externally blinded ones as well. These features, if present, will be a clear indication that the theoretical framework involving non-locality and generalised entanglement is suitable for this line of research. One might argue that synthesis of DNA that is $98 \%$ identical to the original, as Montagnier's group did, makes blinding obsolete. However, the experience acquired through the use of DBCTs in homeopathy and the accumulated data of Benveniste's group, as analysed by Beauvais, indicate that blinding might, per se, be a tool for understanding the underlying phenomena.

\section{Experiments and Clinical Trials Capable of Distinguishing between Local and Non-Local Theories in Homeopathy}

The domain of experimentation with UHDs, let alone the homeopathic medicine, is not characterised by the high degree of mathematical abstraction found in quantum mechanics. Thus, it is perhaps unrealistic to hope to design and conduct a single 'Aspect-type' experiment that will verify or disprove once and for all the non-locality of the underlying theory. ${ }^{17,38}$ Nonetheless, the conjecture that non-locality and generalised entanglement lie at the origin of the homeopathic phenomenon can be tested by various different experimental setups and clinical trials or provings targeting different aspects of existing data.

We have already touched upon several such cases. (1) The articles of Walach et $\mathrm{al}^{28-30}$ (see also references therein) that report specially designed pilot double-blinded pathogenetic trials provide particularly convincing evidence that only a non-local theoretical framework can account for the results. In these articles, the authors mention the shortcoming that was the relatively limited number of provers involved in the performed pilot studies and suggest further refinements of their trial. (2) The experimental work of Thieves et al $^{34}$ offers preliminary support to Beauvais' hypothesis that local blinding increases the effect of a homeopathic medicine on the growth of plants. (3) We have previously suggested that the use of randomly selected homeopathic remedies instead of blank pills as placebo, in a way that excludes future tracing back the identities of those used, might increase the effectiveness of a remedy tested in a double-blinded trial or in suitably designed experimentation with plants or in vitro. ${ }^{32}$ (4) The statistics of multiple repetitions of Montagnier's experiment of DNA synthesis might be suitable to test the applicability of the non-local theoretical framework to experiments of transmission of DNA sequence information from potentised solutions to pure water through electromagnetic signals. As earlier suggested, this would include open as well as internally and externally blinded trials, following the Beauvais model of analysis. ${ }^{16}$ (5) The 'experimenter effect' described in the article reporting the results of the DARPA team on a digital biology experiment ${ }^{35}$ offers an experimental model that seems to be suitable for repetition. It might produce evidence on the impact of the experimenter's influence, something which is in parallel with the conjectured practitioner's involvement in the homeopathic therapeutic entanglement. Finally, in many of the cited articles, the authors have implicitly or explicitly formulated ways to design and conduct experiments that do have the potential to verify or disprove the non-local approach: see, for example, the noted references. ${ }^{8,9,15,26,46}$

\section{Conclusion}

Benveniste's pioneering work and its follow-up, with their often ambiguous outcomes, have challenged some widespread ways of thinking and methodological conventions, 
even among scientists who had worked 'against the stream'. This made them wonder whether something could be wrong, especially those who had played critical roles in this threedecade endeavour. Characteristic of this attitude is the cautious review by Professor M. Ennis, who described herself as 'sceptical'. ${ }^{47}$ This review was followed by an honest and direct response by the author ${ }^{48}$ to a comment of a homeopath criticising her stance. We believe that this sensation of an impasse might be the outcome of the attempt to apply the local biomolecular paradigm (i.e. the conventionally causal and local approach) in homeopathic basic research. However, if this approach is not adequate, it puts experienced researchers into self-doubt because they find the results of their work to be unreliable despite all their efforts.

We have to keep in mind that non-local and local theories about the foundations of the homeopathic phenomenon are not mutually exclusive and they might both be valid, not only in homeopathy but (although in a more subtle way) in conventional medicine as well. ${ }^{11,49}$ Perhaps the homeopathic remedy presents a local component of 'memory'. This might be dependent on clusters of solvent molecules (clathrates), ${ }^{50}$ silicates ${ }^{51}$, active oxygen species, ${ }^{52}$ "coherence domains' formed in water ${ }^{53}$ or combinations of them, depending on the way of preparation of the remedy. Walach ${ }^{8}$ has commented on analogies between quantum teleportation and the homeopathic phenomenon, in the framework of weak quantum theory, ${ }^{25}$ where traces of the initial substance or its 'imprints' constitute a classically conceived information channel.

Differences in the participation of the classical/local component in the homeopathic phenomenon, either in experimentation or in homeopathic treatment, might also exist. These could relate to the well-documented differences in reproducibility between experimental models when working in the range of UHDs. Inhibition of basophil activation by histamine is shown to be a system that often replicates successfully, while direct activation of basophils by anti-IgE is clearly less reproducible. ${ }^{24}$ Reviews of the homeopathic literature are available that focus on experimental models which proved themselves robust to replication. ${ }^{54,55}$ Note also the earlier experiments by Benveniste's group with systems that were particularly stable in replication and were conducted under conditions of randomisation and blinding. ${ }^{56,57}$

The experiments by Benveniste, Ennis and Montagnier (as well as those by other groups, ${ }^{58,59}$ to cite only two other studies involving potentised UHDs), have shown that the conventional 'sceptical' approach that such solutions are 'no more than' pure water, and that homeopathy is simply placebo effect, have to be seriously reconsidered. Perhaps, it is still premature to conclude anything with certainty on the origin of the inconsistency found when attempting to replicate some experiments involving UHDs. We believe that further research is needed, necessitating multi-disciplinary approaches and open-mindedness.

\section{Highlights}

- Non-local explanations for the effects of homeopathy arose from the controversial work of Jacques Benveniste.
- An attempt to demonstrate transmission of the activity of a potentised solution was unsuccessful, but a critical experimenter effect was detected.

- Multiple repetitions of the human basophil degranulation experiments similar to Benveniste's have shown positive results.

- Other workers have proposed non-local hypotheses based on weak quantum theory. These have some experimental support.

\section{Acknowledgments}

We thank Dr Leto Kyritsi for helpful discussions and suggestions during the preparation of this article.

\section{References}

1 Thomas Y. From high dilutions to digital biology: the physical nature of the biological signal. Homeopathy 2015;104:295-300

2 Thomas Y. The history of the memory of water. Homeopathy 2007;96:151-157

3 Poitevin B. The continuing mystery of the memory of water. Homeopathy 2008;97:39-41

4 Poitevin B. Mémoire de l'eau ou présence de la matière? La Revue d'Homéopathie 2011;2:4-11

5 Schiff M. The Memory of Water: Homoeopathy and the Battle of Ideas in the New Science. New York, NY: Harper Thorsons; 1998

6 Beauvais F. Ghosts of Molecules - The Case of the "memory of water". Lulu Press, Collection Mille-Mondes; 2016

7 Walach H. Magic of signs: a non-local interpretation of homeopathy. Br Homeopath J 2000;89:127-140

8 Walach H. Entanglement model of homeopathy as an example of generalized entanglement predicted by weak quantum theory. Forsch Komplementarmed Klass Naturheilkd 2003;10: 192-200

9 Walach H. Entangled-and tied in knots! Practical consequences of an entanglement model for homeopathic research and practice. Homeopathy 2005;94:96-99

10 Milgrom LR. Patient-practitioner-remedy (PPR) entanglement. Part 1: a qualitative, non-local metaphor for homeopathy based on quantum theory. Homeopathy 2002;91:239-248

11 Milgrom LR. The sound of two hands clapping: could homeopathy work locally and non-locally? Homeopathy 2005;94:100-104

12 Milgrom LR. Conspicuous by its absence: the memory of water, macro-entanglement, and the possibility of homeopathy. Homeopathy 2007;96:209-219

13 Weingärtner $\mathrm{O}$. What is the therapeutically active ingredient of homeopathic potencies? Homeopathy 2003;92:145-151

14 Weingärtner 0 . The nature of the active ingredient in ultramolecular dilutions. Homeopathy 2007;96:220-226

15 Weingärtner $\mathrm{O}$. A formal approach to the problem of reproducing experimental effects with homeopathic potencies. J Altern Complement Med 2009;15:287-291

16 Beauvais F. Emergence of a signal from background noise in the "memory of water" experiments: how to explain it? Explore (NY) 2012;8:185-196

17 Beauvais F. A quantum-like model of homeopathy clinical trials: importance of in situ randomization and unblinding. Homeopathy 2013;102:106-113

18 Beauvais F. 'Unconventional' experiments in biology and medicine with optimized design based on quantum-like correlations. Homeopathy 2017;106:55-66

19 Davenas E, Beauvais F, Amara J, et al. Human basophil degranulation triggered by very dilute antiserum against IgE. Nature 1988; 333:816-818

20 Vithoulkas G. The controversy over the "Memory of Water". Medical Science Hypotheses 2017;4:1-6 
21 Belon P, Cumps J, Ennis M, et al. Inhibition of human basophil degranulation by successive histamine dilutions: results of a European multi-centre trial. Inflamm Res 1999;48(Suppl 1):S17-S18

22 Belon P, Cumps J, Ennis M, et al. Histamine dilutions modulate basophil activation. Inflamm Res 2004;53:181-188

23 Maddox J, Randi J, Stewart WW. "High-dilution" experiments a delusion. Nature 1988;334:287-291

24 Sainte-Laudy J, Belon P. Inhibition of basophil activation by histamine: a sensitive and reproducible model for the study of the biological activity of high dilutions. Homeopathy 2009; 98:186-197

25 Atmanspacher $\mathrm{H}$, Römer $\mathrm{H}$, Walach $\mathrm{H}$. Weak quantum theory: complementarity and entanglement in physics and beyond. Found Phys 2002;32:379-406

26 Walach $\mathrm{H}$, Jonas WB, Ives J, van Wijk R, Weingärtner O. Research on homeopathy: state of the art. J Altern Complement Med 2005; 11:813-829

27 Kuhn TS. The Structure of Scientific Revolutions. Chicago and London: University of Chicago Press; 1996

28 Walach H, Sherr J, Schneider R, Shabi R, Bond A, Rieberer G. Homeopathic proving symptoms: result of a local, non-local, or placebo process? A blinded, placebo-controlled pilot study. Homeopathy 2004;93:179-185

29 Möllinger H, Schneider R, Löffel M, Walach H. A double-blind, randomized, homeopathic pathogenetic trial with healthy persons: comparing two high potencies. Forsch Komplementarmed Klass Naturheilkd 2004;11:274-280

30 Walach $\mathrm{H}$, Teut M. Scientific proving of ultra high dilutions on humans. Homeopathy 2015;104:322-327

31 Bayr G. A model for homœopathic drug tests including statistical analysis. Br Homeopath J 1986;75:80-88

32 Almirantis Y. Homeopathy-between tradition and modern science: remedies as carriers of significance. Homeopathy 2013;102: $114-122$

33 Beauvais F. Description of Benveniste's experiments using quantum-like probabilities. J Sci Explor 2013;27:43-71

34 Thieves K, Gleiss A, Kratky KW, Frass M. First evidence of Beauvais' hypothesis in a plant model. Homeopathy 2016;105:270-279

35 Jonas WB, Ives JA, Rollwagen F, et al. Can specific biological signals be digitized? FASEB J 2006;20:23-28

36 Mermin ND. Is the moon there when nobody looks? Reality and the quantum theory. Phys Today 1985;38:38-47

37 Poitevin B. Orientations récentes de la recherche clinique en homéopathie. La Revue d'Homéopathie 2014;5:47-53

38 Fisher P. Entangled, or tied in knots? Homeopathy 2004;93(04): 171-172

39 Lacan J. Écrits: A Selection. New York, NY: W.W. Norton \& Co; 2002

40 Hyland ME, Lewith GT. Oscillatory effects in a homeopathic clinical trial: an explanation using complexity theory, and implications for clinical practice. Homeopathy 2002;91:145-149
41 Olioso D, Marzotto M, Bonafini C, Brizzi M, Bellavite P. Arnica montana effects on gene expression in a human macrophage cell line. Evaluation by quantitative Real-Time PCR. Homeopathy 2016;105:131-147

42 Tsitinidis K. Homeopathic Topology. Hellenic Homeopathic Medical Society (HHMS); 2016. Lon-linearity and Hormesis-like features in homeopathy are discussed throughout the book, and particularly in the appended article "Homeopathy - Therapeutic and Diagnostic Means of Diseases and Bioscience" that firstly appeared in Greek in the Journal of the HHMS "HomeoNews"; Athens, Greece; issue April 2006

43 Montagnier L, Aïssa J, Ferris S, Montagnier JL, Lavallée C. Electromagnetic signals are produced by aqueous nanostructures derived from bacterial DNA sequences. Interdiscip Sci 2009; $1: 81-90$

44 Montagnier L, Aïssa J, Del Giudice E, Lavallee C, Tedeschi A, Vitiello G. DNA waves and water. J Phys Conf Ser 2011;306:012007

45 Montagnier L, Del Giudice E, Aïssa J, et al. Transduction of DNA information through water and electromagnetic waves. Electromagn Biol Med 2015;34:106-112

46 Milgrom LR. Gold standards, golden calves, and random reproducibility: why homeopaths at last have something to smile about. J Altern Complement Med 2009;15:205-207

47 Ennis M. Basophil models of homeopathy: a sceptical view. Homeopathy 2010;99:51-56

48 Ennis M. Reply to D. Mastrangelo. Homeopathy 2010;99:289

49 Fisher P. Local, entangled or both? Homeopathy 2013;102:85-86

50 Anagnostatos GS. Small water clusters (clathrates) in the preparation process of homoeopathy. In: Endler PC, Schulte J, eds. Ultra High Dilution. Springer: Dordrecht; 1994:121-8

51 Anick DJ, Ives JA. The silica hypothesis for homeopathy: physical chemistry. Homeopathy 2007;96:189-195

52 Voeikov VL. The possible role of active oxygen in the memory of water. Homeopathy 2007;96:196-201

53 Bono I, Del Giudice E, Gamberale L, Henry M. Emergence of the coherent structure of liquid water. Water 2012;4:510-532

54 Endler PC, Thieves K, Reich C, et al. Repetitions of fundamental research models for homeopathically prepared dilutions beyond $10^{-23}$ : a bibliometric study. Homeopathy 2010;99:25-36

55 Poitevin B. Survey of immuno-allergological ultra high dilution research. Homeopathy 2015;104:269-276

56 Poitevin B, Davenas E, Benveniste J. In vitro immunological degranulation of human basophils is modulated by lung histamine and Apis mellifica. Br J Clin Pharmacol 1988;25:439-444

57 Davenas E, Poitevin B, Benveniste J. Effect of mouse peritoneal macrophages of orally administered very high dilutions of silica. Eur J Pharmacol 1987;135:313-319

58 Rey L. Thermoluminescence of ultra-high dilutions of lithium chloride and sodium chloride. Physica A 2003;323:67-74

59 Cartwright SJ. Solvatochromic dyes detect the presence of homeopathic potencies. Homeopathy 2016;105:55-65 